\title{
Quantitative electrodiagnostic patterns of damage and recovery after spinal cord injury: a pilot study
}

\author{
Elissa C. Zakrasek ${ }^{1} \cdot$ Jeffrey P. Jaramillo ${ }^{1} \cdot$ Zoia C. Lateva $^{1} \cdot$ Vandana Punj $^{1} \cdot$ B. Jenny Kiratli ${ }^{1} \cdot$ Kevin C. McGill $^{1}$
}

Received: 30 August 2019 / Revised: 16 November 2019 / Accepted: 18 November 2019

This is a U.S. government work and not under copyright protection in the U.S.; foreign copyright protection may apply 2019

\begin{abstract}
Study design Prospective observational pilot study.

Objectives To compare quantitative electromyographic (EMG), imaging and strength data at two time points in individuals with cervical spinal cord injury (SCI).

Setting SCI center, Veterans Affairs Health Care System, Palo Alto, California, USA.

Methods Subjects without suspected peripheral nerve injury were recruited within 3 months of injury. Needle EMG examination was performed in myotomes above, at, and below the SCI level around 11- and 12-months post injury. EMG data were decomposed using custom software into constituent motor unit trains and each distinct motor unit was analyzed for firing rate and amplitude. Strength measurements were made with dynamometry and according to the International Standard of Neurologic Classification of SCI (ISNCSCI). Cervical magnetic resonance images (MRI) were evaluated by two neuroradiologists for gray and white matter damage around the SCI. Here, we compare the EMG, strength, and imaging findings of the one of the four participants who completed both 3- and 12-month EMG evaluations.

Results There was an increase in force generation in all muscles tested at 1 year. Localized findings of very fast firing motor units helped localize spinal cord damage and revealed gray matter damage in spinal segments where MRI was normal. Meanwhile, improvement in strength over time corresponded with different electrophysiologic patterns.

Conclusions Electromyographic decomposition at two time points provides valuable information about localization of spinal cord damage, integrity of motor neuron pools and may provide a unique understanding of neural recovery mechanisms.
\end{abstract}

\section{Introduction}

Electrodiagnostic assessment after spinal cord injury (SCI) is growing in both its clinical and research applications. Type and degree of spinal cord and peripheral nerve damage is predictive of outcome and a detailed understanding of this neurological damage is crucial for the development, refinement, and case-appropriate targeting of regenerative and rehabilitative interventions [1]. electromyographic (EMG) recordings give us a unique ability to tap into the functional dialog between nerves and muscles, unlike neuroimaging and histopathology, which merely give us a structural image of the connection wires.

Elissa C. Zakrasek

Elissa.Zakrasek2@va.gov

1 Spinal Cord Injury Center, VA Palo Alto Health Care System, Palo Alto, CA, USA
SCI affects both upper and lower motor neurons. At the site of injury to the spinal cord, there may be white and/or gray matter damage [2]. Gray matter damage usually involves death of anterior horn cells (AHC) with gradual loss of their axonal projections through Wallerian degeneration [3]. These changes are visualized on electromyography (EMG) as abnormal spontaneous activity (ASA; fibrillation potentials and positive sharp waves), decreased recruitment, and characteristic motor unit (MU) morphologic changes associated with collateral sprouting (polyphasic potentials, increased amplitude, increased duration) [4]. White matter damage at the level of SCI will affect spinal levels and motor neuron pools below that level. Residual motor function and recovery of motor function of muscles innervated by the injured spinal segments therefore depend on the integrity of both the remaining motor neuron pool at the segmental level and the white matter descending tracts above that segment.

There are multiple approaches to EMG that provide varying levels of detail and specificity regarding site and 
degree of injury after SCI. Surface EMG (sEMG) is the most widely used approach as it is noninvasive and accessible to physicians and therapists alike. Unfortunately, sEMG is limited in its ability to detect small signals and cannot effectively localize and differentiate signals, especially those from deep and/or very small muscles [5, 6]. Needle EMG, on the other hand, can more specifically and directly target muscles of interest and may pick up weaker signals missed by sEMG. EMG decomposition is a detailed quantitative analysis that attempts to identify the potentials of the individual MUs in the EMG signal and to measure their morphological and firing-related properties [7-9].

We previously published our EMG decomposition findings in the triceps brachii of four patients with chronic cervical SCI [10]. In that report, we showed both variable denervation patterns between individuals and within different muscle heads of the triceps of the same person. The superfine granularity of detail that can be obtained with EMG decomposition introduces a new way of phenotyping SCI-related weakness that may provide new insight into the underlying distribution of gray and white matter damage. Such detail may then aid in guiding therapeutic intervention and predicting recovery. Further, monitoring such quantitative changes over time may inform our understanding of neural recovery patterns. In the current pilot study, we compare the EMG decomposition findings found at 3 months and 1 year post injury in an individual with acute cervical SCI.

\section{Methods}

Subjects with cervical SCI were recruited from our SCI center if they were within 3 months of initial injury without known or suspected peripheral nerve injury or contraindication to needle EMG. Here, we present data from the one subject who completed both a 3-month and a 1-year EMG assessment.

\section{Clinical assessment}

Results of the International Standard of Neurologic Classification of SCI (ISNCSCI) from the time of admission and around the time of both EMG tests were extracted from the medical record. Lab-based strength assessments were performed on the day of EMG evaluations using a hand-held dynamometer (Lafayette Manual Muscle Test System, Model 01163, Lafayette Instrument CO, Lafayette, IN). The subject's maximum isometric strength was recorded using the "make" test, which measures the maximum force each subject can maintain against resistance supplied by the examiner, expressed in kilograms [11]. Magnetic resonance image (MRI) from the time of injury was not available; however, all subsequent images captured within the first 4 years of injury were reevaluated by two study-related neuroradiologists with specific emphasis on discriminating gray and white matter damage around the level of SCI.

\section{EMG testing}

Needle EMG was performed in muscles innervated by spinal segments above, at, and below the radiographic and clinical level of cord injury in the bilateral arms at $\sim 3$ months and 1 year post injury. The patient sat comfortably in a wheelchair. A disposable concentric needle (TECA Elite, Natus Medical Incorporated, Pleasanton, CA) was inserted in four different sites in each muscle and the patient was asked to perform sustained moderate to strong isometric contractions. The ground electrode was placed close to the insertion site. Ten-second-long signals were amplified (Nicolet Viking, Madison WI) with filter settings of $5 \mathrm{~Hz}$ and $5 \mathrm{kHz}$, sampled at $10 \mathrm{kHz}$, and digitally stored for further analysis. Custom-written software (EMGLAB) was used to decompose each EMG signal into constituent MU trains (for a more detailed discussion of this technique please see reference [8]). Each of the identified distinct MUs was then analyzed for firing rate and amplitude. The firing rate was calculated as a reciprocal mean interdischarge interval over $2 \mathrm{~s}$ sustained MU firing. Although the recordings were $10 \mathrm{~s}$ long, the patient often had difficulty maintaining steady, sustained contraction for the entire duration. The MU amplitude was measured as a peak-topeak amplitude of the averaged MU waveform using the identified firing times as triggers. Strength testing and EMG testing were not always performed in the same muscles, thus direct comparisons of strength and EMG changes were not always possible.

A qualitative estimation of fibrillation potentials and positive sharp waves was performed at the 3- and 12-month assessments by two board certified physiatrists present at the time of the EMG testing. ASA was graded according to Daube [12]. As the patient had no clear cause for peripheral nerve injury, the finding of ASA was ascribed to AHC damage within the gray matter of the spinal cord from primary cord injury or possibly trans-synaptic degeneration [13].

\section{Results}

\section{Clinical assessment}

The subject was a previously healthy 63-year-old man who sustained his SCI from a fall. Neurologic examination revealed an ISNCSCI classification of C4 AIS C at the time of admission to our center. Lab strength testing 
Table 1 Muscle strength.

\begin{tabular}{lllllll}
\hline Level & Muscle & Side & $\begin{array}{l}\text { Force sensor } \\
\text { (3 months) }\end{array}$ & $\begin{array}{l}\text { Force sensor } \\
(12 \text { months })\end{array}$ & $\begin{array}{l}\text { MMT } \\
\text { (3 months) }\end{array}$ & $\begin{array}{l}\text { MMT } \\
\text { (12 months })\end{array}$ \\
\hline C5, 6 & Biceps & R & 5.25 & 23.89 & 2 & 4 \\
C5, 6 & Brachioradialis & R & NT & NT & 1 & 3 \\
C6, 7, 8 & Triceps & R & 0.87 & 3.57 & 1 & 1 \\
C7, 8 & EDC & R & 0.38 & 1.38 & NT & NT \\
C5 & Biceps & L & 5.85 & 18.03 & 2 & 3 \\
C6 & Brachioradialis & L & NT & NT & 3 & 3 \\
C7 & Triceps & L & 0.75 & 8.50 & 1 & 1 \\
C7 & EDC & L & 0.36 & 1.96 & NT & NT \\
\hline
\end{tabular}

Strength measured by force sensor (kg), R, L, MMT graded 1-5, NT

$R$ right, $L$ left, MMT manual muscle test, $N T$ not tested

Table 2 Spinal cord damage at C3/4 injury level.

\begin{tabular}{lll}
\hline PID & MR extent of damage & Description \\
\hline 58 & $0.9 \mathrm{~cm}($ long $) \times 1.0 \mathrm{~cm}$ (transverse $) \times 0.6 \mathrm{~cm}(\mathrm{AP})$ & GM + WM, spared ventral WM \\
373 & $0.9 \mathrm{~cm}($ long $) \times 1.4 \mathrm{~cm}$ (transverse $) \times 0.4 \mathrm{~cm}(\mathrm{AP})$ & Holocord \\
1219 & $1.0 \mathrm{~cm}($ long $) \times 1.3 \mathrm{~cm}$ (transverse $) \times 0.2 \mathrm{~cm}(\mathrm{AP})$ & Holocord, AP diameter reduced atrophy \\
\hline
\end{tabular}

$P I D$ post injury days, $M R$ magnetic resonance image, $G M$ gray matter, $W M$ white matter, $A P$ anterior posterior

and manual muscle testing (MMT) results as performed by his SCI physicians and therapists close to the time of EMG evaluations can be found in Table 1. Detailed neuroradiologist interpretations of the patient's cervical MRIs revealed an injury epicenter at C3-4 with no radiologic evidence of injury at C2, C5, or below (Table 2). Of note, the latter two MRIs revealed an increase in the cross-sectional area of the injury compared with the 2month MRI (Fig. 1).

\section{EMG findings}

All the EMG signals were carefully analyzed using EMGLAB. The ability to perform accurate signal decomposition was limited by the quality of the signal which was often compromised by movement artifact, needle movement, or the patient's inability to produce stable, sustained contractions. Two-second segments from each muscle were carefully selected for firing rate and amplitude calculations. A representative example of the EMG results is shown in Fig. 2.

(1) EMG findings around level of injury:

The trapezius frequently receives motor innervation from branches of $\mathrm{C} 3$ and $\mathrm{C} 4$ and was thus used as possible source of EMG data for these myotomes [14]. The bilateral upper trapezius revealed a normal pattern of MU activation, recruitment, and morphology (as seen in Fig. 2, similar findings seen in the right upper trapezius). EMG of the left medial trapezius and deltoid revealed very fast firing MUs, suggesting gray matter damage at the corresponding left C4-5 spinal levels. Meanwhile, the right levator scapulae revealed a normal EMG decomposition pattern but the right deltoid revealed only two very fast firing MUs (42.0 and 46.6 Hz).

(2) EMG changes vs. strength changes:

Here, we provide detailed commentary only on those muscles for which we had both 3-month and 1-year EMG values and direct or indirect strength assessments at the same time points. Notably, there was an increase in force generation at 1-year compared with the 3-month time point in all cases of laboratory strength testing. Concurrent changes in gross MMT on ISNCSCI were not always seen (Table 1). Meanwhile, EMG decomposition revealed variable patterns of change between the two EMG time points as described below and seen in Fig. 3.

\section{Biceps brachii (C5-6)}

Bilateral biceps brachii demonstrated increased strength on MMT and laboratory force testing. ASA (2-3+) was noted at the time of initial EMG assessment only (Table 3). EMG decomposition on the left revealed similar firing rates (all between 6 and $8 \mathrm{~Hz}$ ) with only two MUs at both time points and increased amplitudes seen at 1 year. The right revealed 
Fig. 1 MRI images at three time points. Axial and sagittal T2 MRI images from the injury epicenter at (a) 58 days post injury, (b) 373 days post injury, and (c) 1219 days post injury.
A.
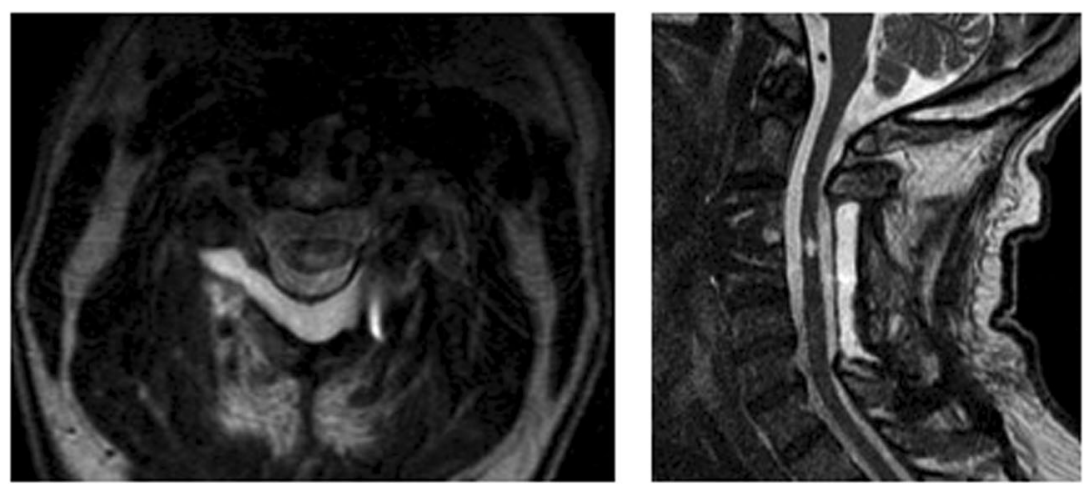

B.
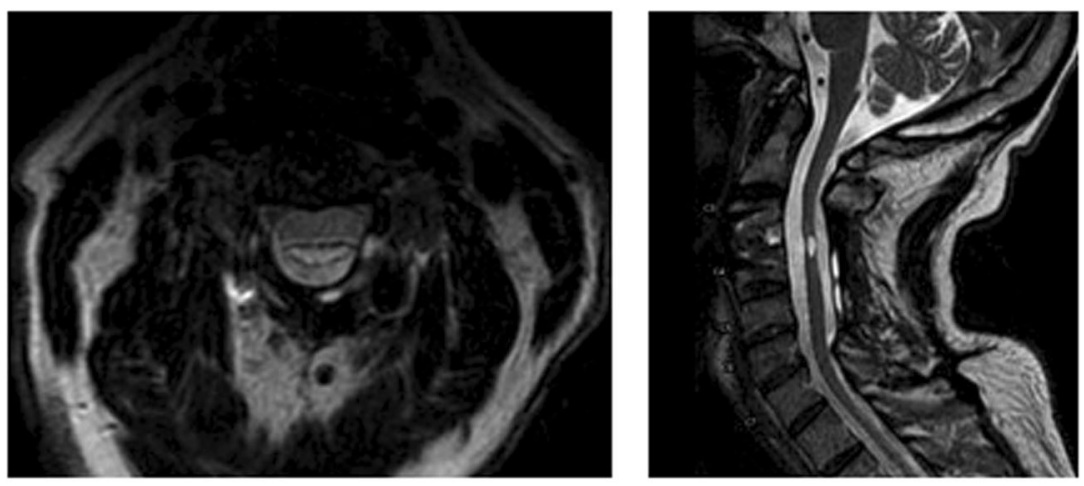

C.

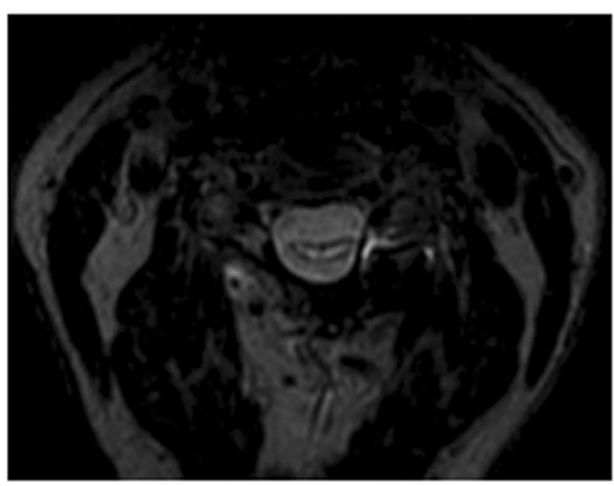

one very fast firing $\mathrm{MU}$ at both time points (44.7 and $50.9 \mathrm{~Hz}$ ) but increased MU amplitudes were seen at 1 year.

\section{Brachioradialis (C5-6)}

There was no direct strength testing of the brachioradialis; however, ISNCSCI examination did reveal increased strength in the right C6 myotome with stable left C6 myotome strength. ASA $(2-3+)$ was noted at the time of initial EMG assessment only (Table 3). EMG decomposition on the left revealed few MU's with similar firing rates (all between 4 and $8 \mathrm{~Hz}$ ) at both time points but increased amplitudes at 1 year. The right revealed a mixed pattern of firing rates at both time points $(6.7-47.1 \mathrm{~Hz}$ then $6.0-54.3 \mathrm{~Hz})$ with faster firing MUs demonstrating increased amplitudes at 1 year.
Triceps brachii $(C 6,7,8)$

The bilateral triceps brachii demonstrated increased strength on laboratory force testing but not on MMT. Significant ASA (3+) was only seen on the right at 3 months however there was $1+$ ASA seen on the left at 1 year (Table 3). EMG decomposition on the left revealed MUs with similar firing rates $(7.3-8.9 \mathrm{~Hz}$ then $6.1-6.7 \mathrm{~Hz})$ and a narrowed range of amplitudes at 1 year. The right revealed MUs with similar firing rates at both time points (all between 5.6 and $9.5 \mathrm{~Hz}$ ) and similar amplitudes seen at 1 year.

\section{Extensor digitorum communis (EDC) (C7-8)}

There was no direct strength testing of the EDC and ISNCSCI examination revealed no change of strength in the $\mathrm{C} 7$ 

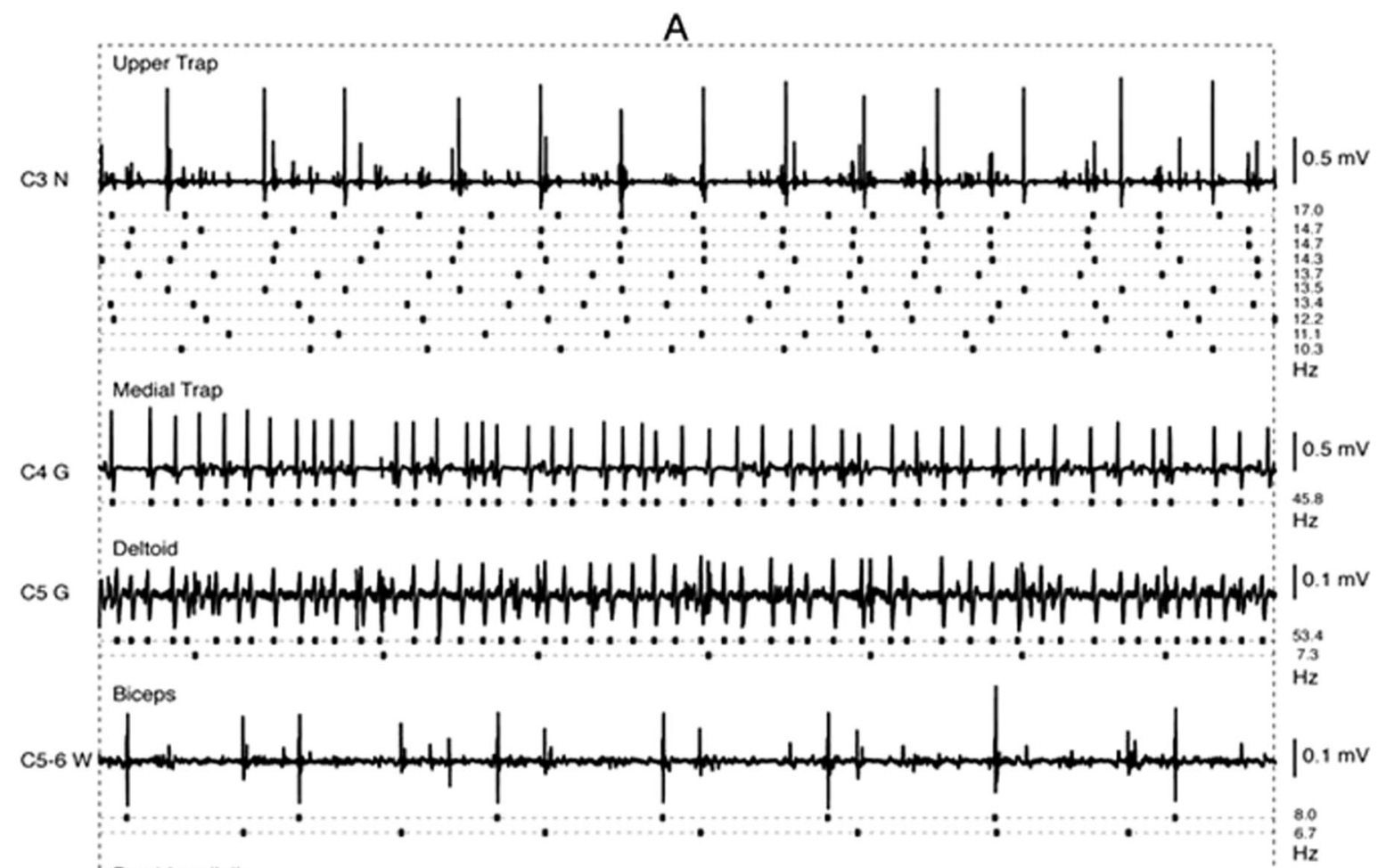

C6 $\mathrm{w}$

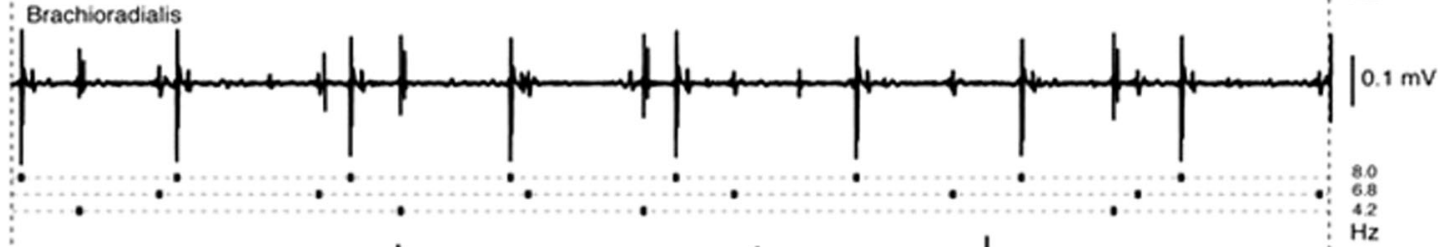

C6 W

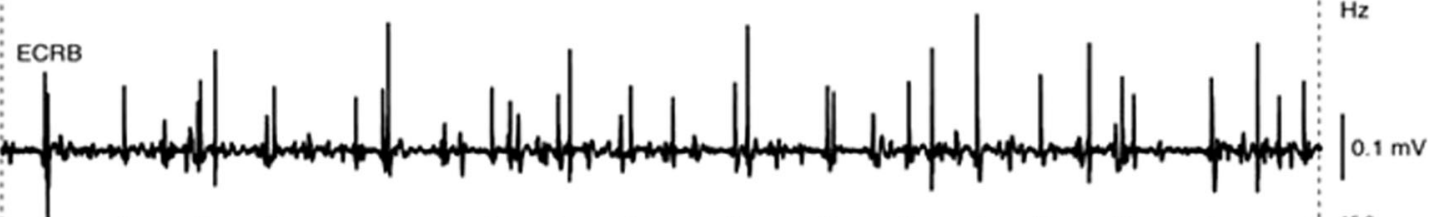

C7 w
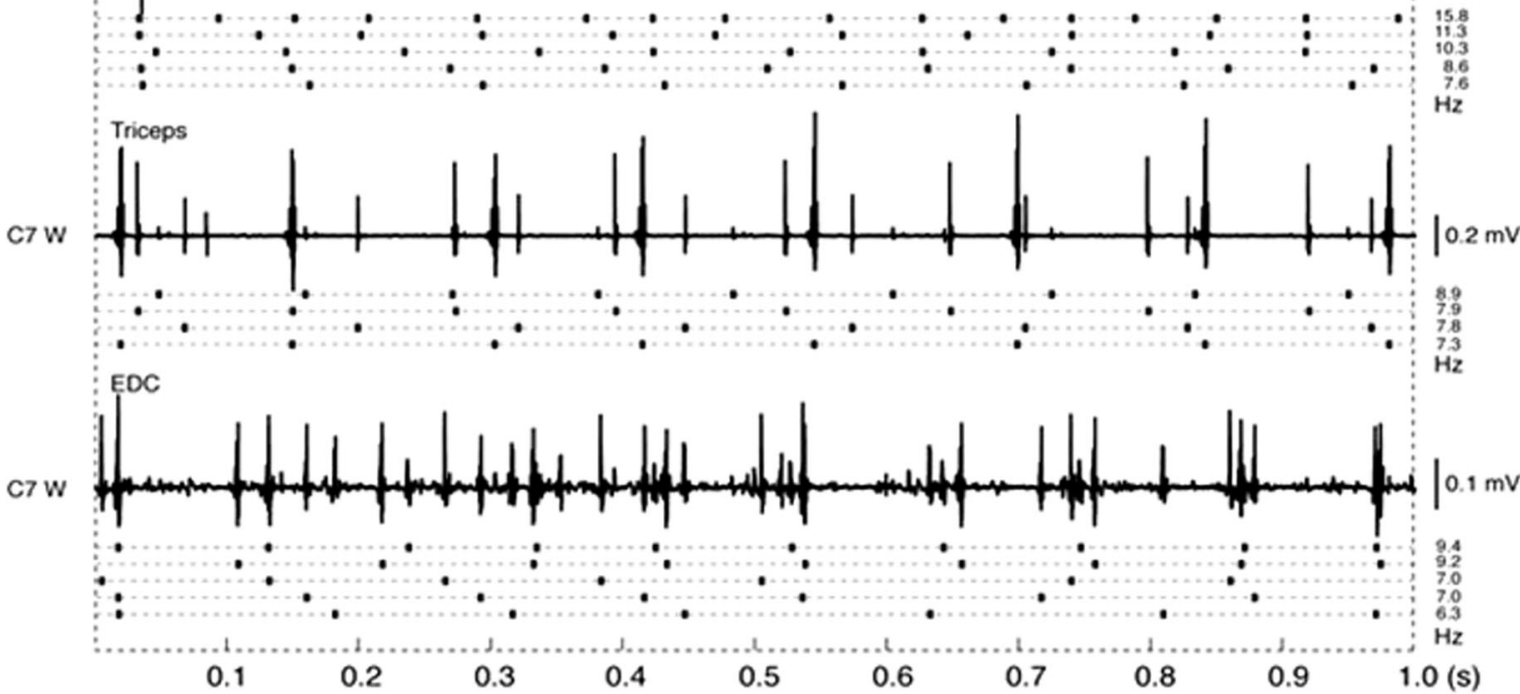

myotome. ASA (3+) was only seen on the left at 3 months (Table 3). EMG decomposition on the left revealed MUs with similar firing rates $(6.3-9.4 \mathrm{~Hz}$ then $5.8-14.3 \mathrm{~Hz})$ but lower amplitudes. The right revealed few MUs at both time points with similar firing rates (all between 6.8 and $10.1 \mathrm{~Hz}$ ) but increased amplitudes at 1 year. 
Fig. 2 EMG decomposition 3 months post injury. Example of the EMG results from the left side at 3 months. For each muscle tested, the upper trace shows 1s-long EMG signal. Below it, the identified firing times of each individual motor unit (in a line) are shown as dots. The mean firing rate for each motor unit are shown to the right. Here the upper trapezius represents a normal interference pattern with ten active motor units discharging with mean rates between 10.3 and $17.0 \mathrm{~Hz}$. Only 1 and 2 motor units were recorded in the medial trapezius and deltoid, respectively. In these muscles, the mean firing rates were calculated as high as 45.8 and $53.4 \mathrm{~Hz}$ with maximum instantaneous rate up to $90 \mathrm{~Hz}$. In the biceps brachii, brachioradialis, triceps brachii, and extensor digitorum communis muscles, motor units were discharging regularly, but the mean firing rates were all below $10 \mathrm{~Hz}$ and as low as $4.2 \mathrm{~Hz}$. Firing rate $(\mathrm{Hz})$, microvolt $(\mathrm{mV})$, seconds(s).

\section{Discussion}

Three notable observations are made from the findings detailed above: (1) EMG helps delineate a microscopic level of injury, an "electrodiagnostic level of injury," especially in myotomes where strength testing is not routinely done, (2) there is electrodiagnostic evidence suggestive of gray matter damage at spinal levels below the radiographic level of injury, (3) clinical improvements in strength may correlate with different patterns of electrodiagnostic change

\section{(1) Electrodiagnostic level of injury:}

At the radiographic level of SCI in this case (C3-4), strength testing is not routinely done and concurrent cranial nerve innervation may confound patterns of motor preservation. Here we saw different patterns of EMG findings between adjacent motor neuron pools of the left upper and medial trapezius, suggesting a highly granular localization of the injury level. These findings suggest a left-sided "electrodiagnostic level of injury" localized between the motor pools of the upper and medial trapezius while the right-sided electrodiagnostic level of injury lies between the motor neuron pools for the levator scapula and deltoids. EMG thus offered an alternative view of the injury level not easily appreciated clinically. Further, our EMG findings suggest that one might be able to electrodiagnostically define both the upper and lower bounds of direct cord damage as well as the extent and type of injury there within.

(2) Gray matter damage below radiographic injury:

As noted, injury to the spinal cord was visualized radiographically at C3-4 without significant cord damage perceived radiographically below this level. Meanwhile, there was EMG evidence of gray matter damage in the C5-6 myotome, and possibly even lower. This is consistent with Berman et al. who showed electrodiagnostic evidence of denervation
$2-5$ segments below the clinically and radiographically defined level of cord injury [15]. In the C5-6 myotome, MU amplitudes were generally higher at the 1-year time point, which could represent maturation of collateral sprouting following AHC loss at the time of initial injury. This finding of increased MU amplitudes was also seen in our prior work [10]. The nearly ubiquitous finding of ASA at 3 months that resolved by 1 year is also consistent with initial denervation at these levels. Finally, the very fast firing MUs observed in C5-6 innervated muscles also likely reflect motor neuron loss. We saw MU firing rates above $45 \mathrm{~Hz}$, which is higher than the maximum sustained firing rates observed in comparable muscles of able-bodied subjects (biceps brachii: $21-31$ range $15-41 \mathrm{~Hz}$ ) [2, 16]. One of the few other conditions in which such high firing rates have been reported is postpolio syndrome [17].

In contrast, muscles innervated by spinal segments farther from the primary cord damage site were more likely affected by impaired descending activation secondary to white matter damage rostrally (i.e., upper motor neuron injury). Unlike in the C5-6 myotome, no very fast firing MUs were observed in these muscles. Of note, we do not attempt to define a lower limit of normal for MU firing rate in this study and lower firing rates should be interpreted with caution in this report as the patient performed submaximal contractions. Nonetheless, the work of Zijdewind and Thomas suggests that steady, sustained, slow firing rates between 4 and $6 \mathrm{~Hz}$, as we saw in some muscles, are not normally observed $[18,19]$. Finally, the inconsistent findings of ASA in the C7-8 myotomes may also suggest less gray matter damage; however, evaluation of spontaneous activity is a subjective assessment which does not always correlate with degree of denervation [2]. Indeed, the meaning of fibrillation potentials and positive sharp waves after SCI is a debated topic. These findings may indicate muscle denervation secondary to spinal cord gray matter damage, other peripheral nerve injury, or compromise of the neuromuscular junction [20]. Even AHC loss may be directly or indirectly attributable to the SCI, according to the theory of trans-synaptic degeneration.

\section{(3) Variable recovery patterns:}

Force data were directly comparable with EMG data for the biceps and triceps. In both muscles, there was an increase in force generation noted at 1 year, more pronounced in the biceps than the triceps and only perceivable on MMT in the biceps. While strength improved, EMG changes over time were different in each muscle. In the biceps, very few MUs were able to be elicited bilaterally at the 1-year assessment but those elicited had high amplitudes and the right biceps still 
Fig. 3 Motor unit firing rates and amplitudes EMG decomposition of motor units of firing rates and amplitudes for four muscles; biceps, brachioradialis, extensor digitorum communis (EDC), and triceps. Muscles for left (L) and right $(\mathrm{R})$ upper extremities for 3 months (T1) and 12 months (T2) post injury time points. Firing rate $(\mathrm{Hz})$, amplitude (AMP), microvolt $(\mathrm{mV})$.

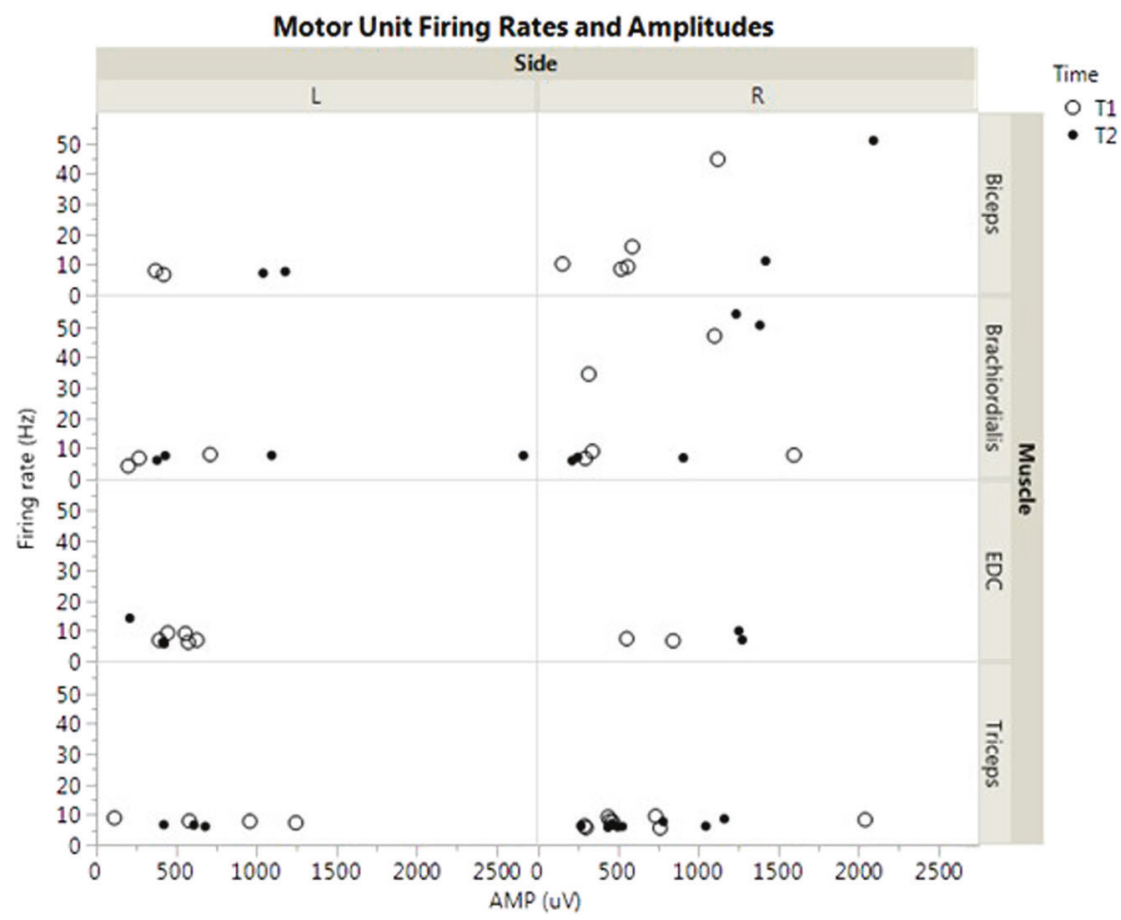

Table 3 Spontaneous activity.

\begin{tabular}{llllll}
\hline & & \multicolumn{3}{l}{ Fibrillation scale values } \\
\cline { 3 - 6 } Spinal level & Muscle & Right (3 months) & Right (12 months) & Left (3 months) & Left (12 months) \\
\hline C5, 6 & Biceps brachii & $3+$ & 0 & $2+$ & 0 \\
C5, 6 & Brachioradialis & $2+$ & 0 & $3+$ & 0 \\
C6, 7, 8 & Triceps & $3+$ & 0 & 0 & $1+$ \\
C7, 8 & EDC & 0 & 0 & $3+$ & 0 \\
\hline
\end{tabular}

Abnormal spontaneous activity scale $(0=$ absent, $1+=$ fibrillation potentials and/or positive sharp waves persistent in at least 2 areas, $2+$ : moderate number of persistent fibrillation potentials and/or positive sharp waves in 3 or more areas, $3+$ : large number of persistent discharges in all areas, 4+: profuse, widespread, persistent discharges that fill the baseline)

had a very fast firing MU. In the triceps, firing rates and amplitudes were similar over time. These findings demonstrate how improved strength results from different mechanisms depending on the underlying pathology and may be multifactorial resulting from axonal sprouting, reversal of disuse atrophy, resolution of pain inhibition, neuroplasticity of spared pathways, and functional training [21]. Little et al. noted the variability in electrophysiologic patterns corresponding to upper limb weakness in a series of 22 people with tetraplegia, but did not repeat these assessments over time or correlate electrophysiologic changes with strength changes [2]. Greater numbers of case examples will be needed to better delineate the time course of these changes and to better understand the prognostic value of these variable findings.

\section{Limitations}

Of note, our interpretation of the EMG decomposition data is limited by numerous factors. First, intramuscular EMG only samples a small cross section of each muscle thus we cannot guarantee that all motor resources were represented. We tried to minimize this limitation by sampling four sites per muscle. Second, MU amplitude assessment is affected by the proximity of needle placement relative to the MU in question. MUs with increased innervation ratio as a result of sprouting can be detected by an electrode further from the MU territory. Therefore, it is possible that our results underestimated some of the true MU amplitudes. Third, it is impossible to sample the same MUs at two time points, thus sampling a different subset of MUs may occur at each assessment. Fourth, we have intentionally made no efforts to define cutoffs for high and low MU firing rates for this case but did comment on likely deviations above and below expected firing frequencies. Lastly, although there was no clinical suspicion or radiographic evidence for peripheral nerve injury (such as radiculopathy or plexopathy), it is possible that unknown secondary peripheral nerve injury was present. We notably did not include assessment of ulnar or median nerve 
innervated hand muscles which are frequently affected by peripheral compression neuropathies [22, 23].

Many of the limitations above are true of most needle EMG studies as it is neither feasible nor ethical to insert the electrode in every small segment of a muscle. Despite these limitations, we are confident that this data offers useful information about the general state of the spinal segment corresponding to the muscles tested, information that cannot be obtained in other ways. Overall it will be important to repeat this work in a larger sample of people with SCI to ensure reproducibility of findings and to better interpret EMG patterns in the context of clinical findings and prognosis.

\section{Conclusion}

This case study is the first report of quantitative EMG assessments done at two time points after acute SCI. While these findings could be interpreted and debated in different ways and should ultimately be evaluated in the context of numerous other case examples, we are encouraged that this EMG decomposition technique provides valuable information about the type and degree of spinal cord damage and may provide insight into neural recovery mechanisms when performed repeatedly over time. Given that current and future therapeutic neuroregenerative strategies do and will depend upon the type (white vs. gray) and degree of cord damage, these findings reenforce the idea that EMG supplements imaging and clinical exam in understanding spinal cord damage. Further study will help us find clues within these patterns which will continue to guide prognostication and treatment after SCI.

Acknowledgements We would like to thank the individual veterans for their participation in this study. The authors would like to give special thanks to Michelle Nguyen, MD for her high-quality work interpreting the magnetic resonance images for this study.

Funding This study was funded by the Department of Defense SC130314.

\section{Compliance with ethical standards}

Conflict of interest The authors declare that they have no conflict of interest.

Publisher's note Springer Nature remains neutral with regard to jurisdictional claims in published maps and institutional affiliations.

\section{References}

1. Hubli M, Kramer JLK, Jutzeler CR, Rosner J, Furlan JC, Tansey $\mathrm{KE}$, et al. Application of electrophysiological measures in spinal cord injury clinical trials: a narrative review. Spinal Cord. 2019. https://doi.org/10.1038/s41393-019-0331-z.

2. Little JW, Powers RK, Michelson P, Moore D, Robinson LR, Goldstein B. Electrodiagnosis of upper limb weakness in acute quadriplegia. Am J Phys Med Rehabil. 1994;73:15-22.

3. Becerra JL, Puckett WR, Hiester ED, Quencer RM, Marcillo AE, Post MJ, et al. MR-pathologic comparisons of wallerian degeneration in spinal cord injury. AJNR Am J Neuroradiol. 1995;16:125-33.

4. Preston DC, Shapiro BE. Needle electromyography. Fundamentals, normal and abnormal patterns. Neurol Clin. 2002;20:361-96.

5. Skinner SA, Transfeldt EE, Savik K. Surface electrodes are not sufficient to detect neurotonic discharges: observations in a porcine model and clinical review of deltoid electromyographic monitoring using multiple electrodes. J Clin Monit Comput. 2008;22:131-9.

6. Hogrel J-Y. Clinical applications of surface electromyography in neuromuscular disorders. Neurophysiol Clin. 2005;35:59-71.

7. Stashuk D. EMG signal decomposition: how can it be accomplished and used? J Electromyogr Kinesiol. 2001;11:151-73.

8. McGill KC, Lateva ZC, Marateb HR. EMGLAB: an interactive EMG decomposition program. J Neurosci Methods. 2005;149:121-33.

9. Parsaei H, Stashuk DW, Rasheed S, Farkas C, Hamilton-Wright A. Intramuscular EMG signal decomposition. Crit Rev Biomed Eng. 2010;38:435-65.

10. Johanson ME, Lateva ZC, Jaramillo J, Kiratli BJ, McGill KC. Triceps brachii in incomplete tetraplegia: EMG and dynamometer evaluation of residual motor resources and capacity for strengthening. Top Spinal Cord Inj Rehabil. 2013;19:300-10.

11. Bohannon RW. Make tests and break tests of elbow flexor muscle strength. Physiother Can 1990;42:247-51.

12. Daube JR. AAEM minimonograph \#11: Needle examination in clinical electromyography. Muscle Nerve. 1991;14:685-700.

13. Spielholz NI, Sell GH, Goodgold J, Rusk HA, Greens SK. Electrophysiological studies in patients with spinal cord lesions. Arch Phys Med Rehabil. 1972;53:558-62.

14. Kim JH, Choi KY, Lee KH, Lee DJ, Park BJ, Rho Y-S. Motor innervation of the trapezius muscle: intraoperative motor conduction study during neck dissection. ORL J Otorhinolaryngol Relat Spec. 2014;76:8-12.

15. Berman SA, Young RR, Sarkarati M, Shefner JM. Injury zone denervation in traumatic quadriplegia in humans. Muscle Nerve. 1996;19:701-6.

16. Bellemare F, Woods JJ, Johansson R, Bigland-Ritchie B. Motorunit discharge rates in maximal voluntary contractions of three human muscles. J Neurophysiol. 1983;50:1380-92.

17. Larsson L, Li X, Tollbäck A, Grimby L. Contractile properties in single muscle fibres from chronically overused motor units in relation to motoneuron firing properties in prior polio patients. $\mathrm{J}$ Neurol Sci. 1995;132:182-92.

18. Thomas CK, Broton JG, Calancie B. Motor unit forces and recruitment patterns after cervical spinal cord injury. Muscle Nerve. 1997;20:212-20.

19. Zijdewind I, Thomas CK. Motor unit firing during and after voluntary contractions of human thenar muscles weakened by spinal cord injury. J Neurophysiol. 2003;89:2065-71.

20. Riley DA, Burns AS, Carrion-Jones M, Dillingham TR. Electrophysiological dysfunction in the peripheral nervous system following spinal cord injury. PM R. 2011;3:419-25.

21. DeLisa JA, Little JW. Electrodiagnosis and recovery of function. Am J Phys Med Rehabil. 1988;67:44-9.

22. Tun CG, Upton J. The paraplegic hand: electrodiagnostic studies and clinical findings. J Hand Surg. 1988;13:716-9.

23. Nogajski JH, Engel S, Kiernan MC. Focal and generalized peripheral nerve dysfunction in spinal cord-injured patients. J Clin Neurophysiol. 2006;23:273-9. 